\title{
Zum Tode von Adolf Moxter
}

\author{
Wolfgang Ballwieser
}

Online publiziert: 31. Mai 2018

(C) Schmalenbach-Gesellschaft für Betriebswirtschaft e.V. 2018

Am 7. April 2018 starb Professor Dr. Dr. h.c. mult. Adolf Moxter in Bad Soden am Taunus, wo er seit dem Sommer 2006 mit seiner Gattin lebte. Die universitäre Landschaft verliert mit ihm einen gleichermaßen herausragenden wie angesehenen Forscher und Lehrer auf dem Gebiet der Betriebswirtschaftslehre, speziell der Rechnungslegung und Unternehmensbewertung, der mit seinem Wirken weit über seine Heimatuniversität und die BWL hinausstrahlte.

Adolf Moxter wurde am 3. Oktober 1929 als Sohn einer Gärtnerfamilie in Frankfurt am Main geboren. Er wuchs in Bockenheim auf, jenem Stadtteil, in dem bis vor wenigen Jahren das Zentrum der Johann Wolfgang Goethe-Universität lag. Hier studierte er ab Sommersemester 1950 Betriebswirtschaftslehre und wurde nach dem Diplomexamen im Jahr 1954 Assistent von Karl Hax. Schon zwei Jahre später beendete er seine Doktorarbeit, die 1957 unter dem Titel „Methodologische Grundfragen der Betriebswirtschaftslehre" veröffentlicht wurde. Zehn Jahre später erschien diese Arbeit in japanischer Übersetzung. 1961 habilitierte er sich - u. a. nach einem Aufenthalt in Großbritannien - mit einer von Karl Hax und dem Volkswirt Heinz Sauermann begutachteten Arbeit über den Einfluss von Publizitätsvorschriften auf das unternehmerische Verhalten. Diese im Folgejahr publizierte Arbeit war aufgrund ihrer mikroökonomischen Ausrichtung ihrer Zeit weit voraus. Unmittelbar nach der Habilitation folgte er dem Ruf auf eine ordentliche Professur für Betriebswirtschaftslehre an der Universität des Saarlandes. Die Frankfurter Universität berief ihn 1965 zurück. Dort übernahm er einen von der Metallgesellschaft gestifteten Lehrstuhl für Betriebswirtschaftslehre und wurde Direktor des Seminars für Treuhandwesen, dem er trotz ehrenvoller und verlockender Rufe treu blieb. In den Jahren 1967/68

W. Ballwieser $(\square)$

Fakultät für Betriebswirtschaft, Ludwig-Maximilians-Universität, München, Deutschland

E-Mail: ballwieser@bwl.lmu.de 
war er zu schwierigster universitärer Zeit erfolgreicher Dekan der damaligen Fakultät.

Die Universität Trier, die Ludwig-Maximilians-Universität München und die Universität Leipzig haben die großen wissenschaftlichen Leistungen von Adolf Moxter in den Jahren 1992 (Trier und München) und 1999 (Leipzig) mit Ehrenpromotionen gewürdigt. Das Institut der Wirtschaftsprüfer ernannte ihn 1991 zum Ehrenmitglied. Der Kommission Rechnungswesen des Verbands der Hochschullehrer für Betriebswirtschaft und dem Ausschuss für Unternehmensrechnung im Verein für Socialpolitik gehörte er über Jahrzehnte hinweg an. Im Jahr 1994 wurde ihm eine Festschrift unter dem Titel „Bilanzrecht und Kapitalmarkt“ überreicht, die in mehr als 60 Beiträgen auf 1500 Seiten seinen Einfluss auf Wissenschaft, Praxis und Rechtsprechung dokumentiert. Anlässlich der Emeritierung hielt er am 22. Oktober 1999 nach treffender Laudatio des Dekans Heinz Isermann seine Abschiedsvorlesung zum Thema „Rechnungslegungsmythen“. Sie ist im Oktober 2000 in erweiterter Form in der Zeitschrift „Betriebs-Berater“ erschienen. Würdigungen seiner Person, seiner Forschung und Lehre erfolgten aus der Feder einiger seiner Schüler zum 60. Geburtstag in der ZfbF, zum 70. Geburtstag in der Deutschen Steuer-Zeitung und zum 80. Geburtstag in der Wirtschaftsprüfung. Michael Hommel und Thomas Berndt widmeten ihm 2009 einen Beitrag im „Betriebs-Berater“ über „,Das Realisationsprinzip - 1884 und heute“. Unter demselben Titel hatte der Geehrte 1984 publiziert.

Als junger Forscher widmete sich Adolf Moxter der Wechselwirkung zwischen Investitions- und Bilanztheorie in einer Serie großer Aufsätze in der ZfbF, die bereits zu seiner Berühmtheit beitrugen. Es ging ihm insbesondere um die Grenzen der Leistungsfähigkeit einer Bilanz und GuV. Mit der ersten Auflage seines Lehrbuchs „Bilanzlehre“ im Jahr 1974 trat keine Relativierung dieser Grenzen ein, er setzte sich vielmehr nun intensiver mit ihrer Rechtsgrundlage auseinander. Das Buch entstand in der für ihn typischen Arbeitsweise: Wöchentlich abgelieferte Diktate wurden am Lehrstuhl mit Schreibmaschine geschrieben, zuhause redigiert und die Gesamtergebnisse anschließend in einem dicken Manuskript den Mitarbeitern mit der Bitte um Anmerkungen zugänglich gemacht. Hier war so gut wie nichts mehr zu korrigieren, schon gar nichts zu ergänzen. Es gab nur so geringfügige Änderungswünsche wie beispielsweise den, er möge aus dem in einer Aufgabe erwähnten Fußballstadion in Offenbach lieber „Bieberer Berg“ machen. Adolf Moxter war mit anderen Worten nahezu vollkommener „Einzelkämpfer“, was sich auch in den nur ganz wenigen Publikationen mit Mitarbeitern zeigt. Dies entsprach seinem Arbeitsethos und seiner verinnerlichten Aufgabe und Verantwortung als Hochschullehrer.

Die Bilanzlehre erfuhr - wie alle seine Lehrbücher - mehrere Auflagen (2. Aufl. 1976, 3. Aufl. 1984 und 1986), die eine weitere Eigenschaft von ihm kennzeichnen: die jeweils nahezu vollständige inhaltliche Überarbeitung, keineswegs nur durch Teilung, hier von der zweiten auf die dritte Auflage. Das Bilanzrecht ließ Adolf Moxter nicht los. Seinem im Herbst 1981 abgeschlossenen und 1982 publizierten Werk „Betriebswirtschaftliche Gewinnermittlung“ folgte die ebenfalls 1982 veröffentlichte erste Auflage von „Bilanzierung nach der Rechtsprechung des Bundesfinanzhofs“, die Grundlage der von ihm so genannten Bilanzrechtstheorie werden sollte. Die 
zweite bis sechste Auflage des letztgenannten Buchs (1985, 1993, 1996, 1999 und 2007 publiziert) erschien unter dem griffigeren Titel „Bilanzrechtsprechung“.

Die Neuorientierung des früher der Investitions- und Finanzierungstheorie wie der betriebswirtschaftlichen statt handels- und steuerrechtlichen Gewinnermittlung zugeneigten Adolf Moxter verstand nicht jeder Kollege, erst recht dann nicht, wenn er sich beispielsweise an vermeintlich ökonomischer Analyse des Bilanzrechts mit anderen Methoden orientierte. Wahrscheinlich übersah der Kollege dann, dass Adolf Moxter in der Lage war, ein in sich stimmiges System von mit der Rechtsprechung des BFH zu vereinbarenden Bilanzrechtsprinzipien (abgekürzt als Grundsätze ordnungsmäßiger Buchführung oder GoB bezeichnet) zu entwickeln und dort, wo es an Stimmigkeit fehlte, die Bruchstellen aufzuzeigen und Ratschläge zur Verbesserung zu geben. Im Gespräch und in der Auseinandersetzung mit Georg Döllerer, der 1959 deduktiv (,durch Nachdenken“) zu gewinnende GoB verlangte, und mit Heinrich Beisse sowie zahlreichen anderen großen Richterpersönlichkeiten des BFH entwickelte er die Lehre von der Bilanz im Rechtssinne als Ausdruck der wirtschaftlichen Betrachtungsweise des Bilanzrechts. Er hat hierdurch - neben der Gesetzgebung auch die Rechtsprechung des BFH mit beeinflusst. Seine im Jahr 2003 erschienenen „Grundsätze ordnungsgemäßer Rechnungslegung“ atmen denselben Geist, gehen jedoch auch darüber hinaus, was u. a. die Rezensionen von Christoph Kuhner in der ZfB und Jochen Schreiber in der NJW, beide 2004, verdeutlichen.

Ein zweites großes Arbeitsgebiet in den rd. 200 Veröffentlichungen von Adolf Moxter betrifft die Unternehmensbewertung. Hier legte er 1976 die erste und 1983 die zweite Auflage der „Grundsätze ordnungsmäßiger Unternehmensbewertung“ vor, die bis heute in Inhalt, Tiefe und stilistischer Prägnanz ihresgleichen sucht und keineswegs überholt ist. Sein in der zweiten Auflage auf S. $123 \mathrm{zu}$ findender Satz „Bewerten heißt vergleichen (...)“ wurde Allgemeingut von Veröffentlichungen zur Unternehmensbewertung, gelegentlich mit verkehrter Schreibweise oder ohne Quellenangabe. Auf diesem Gebiet hat er zudem in zahlreichen Aufsätzen pointiert und engagiert Stellung bezogen, stets theoretisch versiert und bestens verständlich. Wenn er es in jüngerer Zeit nicht mehr vertieft hat, war dies allein seiner Konzentration auf die Bilanzrechtstheorie geschuldet.

Die im Oktober 2000 erschienene Abschiedsvorlesung war keineswegs das Ende der Veröffentlichungen von Adolf Moxter. Zahlreiche Zeitschriftenaufsätze zur Bilanzrechtsprechung, zu nationaler und internationaler Rechnungslegung, Festschriftbeiträge für Winfried Mellwig, Helmut Laux, Welf Müller und mich sowie Rezensionen zu Bilanzrechtskommentaren erschienen in kurzem zeitlichen Abstand. Das passt zu der Tatsache, dass zu seinem Todeszeitpunkt die überarbeitete Auflage der „Grundsätze ordnungsgemäßer Rechnungslegung“ fertiggestellt war und Mitte des Jahres zum Verlag gegeben werden sollte.

Der Einfluss von Adolf Moxter auf Theorie und Praxis ist kaum zu überschätzen. Weit über hundert Doktoranden wurden von ihm betreut und haben ihre Monographien in renommierten Verlagen publiziert. Wenn ich es recht sehe, finden sich zwanzig Professoren aus seinem Schülerkreis an Universitäten und Fachhochschulen. Tausende von Studenten hat er durch seine unnachahmliche Art, Vorlesungen und Seminare zu bestreiten, für sich und seine Thesen eingenommen. Die von ihm Ausgebildeten haben zum Teil höchste Positionen - nicht nur in Wirtschaftsprü- 
fungsgesellschaften oder Industrieunternehmen - erklommen und unterschwellig oder bewusst das bei und von ihm Erlernte angewendet. Dabei ging es nicht oder nicht vorrangig um Faktenwissen, sondern um die Bewältigung von Problemen durch angestrengte geistige Arbeit. An der Universität gab es unter seinen Zuhörern keine Unentschlossenen: Man fand entweder seine Art und seine Veranstaltungen herausragend und wurde zum „Fan“ oder man wandte sich schnell seinen Kollegen zu. Die erste Gruppe war in großer Überzahl.

Hinzu kamen, neben den Auseinandersetzungen mit seinen Thesen an anderen Hochschulen, seine Tätigkeiten als langjähriger Aufsichtsratsvorsitzender einer WPGesellschaft, als Vorstand wichtiger Stiftungen, als geladener Sachverständiger zu Gesetzesvorhaben, als Gutachter in Rechtsstreitigkeiten oder als Mitglied informeller Gruppen wie der mit dem Namen seines Freundes Hermann Clemm verbundenen Runde. Das machte ihn nicht zum „Manager“ oder „Politiker“. Sein Herz gehörte der Wissenschaft mit der Suche nach Wahrheit, der Verbesserung von Theorie und der Ausbildung seiner Zuhörer. Mehr als eindrucksvoll war seine große Bereitschaft, für ganz junge Semester, bei denen er Entwicklungspotential erkannte, (oftmals umständliche und aufwendige) Anträge auf Förderung mit Stipendien zu stellen oder sie an seinen Lehrstuhl zu binden. Ohne eigene Kinder zu haben, fand er sie im übertragenen Sinne bei den Mitarbeitern, denen er maximalen Freiraum ließ, nicht ohne sie gelegentlich direkt oder indirekt zu lenken.

Adolf Moxter hatte stets ein offenes Ohr für Neuigkeiten und Probleme seiner Mitarbeiter und förderte sie, wo und wie er konnte. Das bedeutete nicht, dass er etwa zu einem gemeinsamen Mittagessen schritt. Er hielt Distanz, ohne im Geringsten unfreundlich zu sein. Er erschien bei Hochzeiten von Mitarbeitern oder Begräbnissen aus deren Familienkreis, schickte Bücher zum Geburtstag und Bethmännchen zu Weihnachten in fremde Orte. Seine wohldurchdacht und brillant formulierten Briefe waren so eindrucksvoll, dass man sie aufhob. Nichtsdestoweniger trat er bei allem mit kaum zu überbietender Bescheidenheit auf, zeigte bewusst nicht viel von sich selbst. Bekannt war, dass er am liebsten französische Philosophen las und mit Goethe „auf Du und Du“ stand. Ausfluss dieser Eigenschaften waren nicht nur sein herausragender Stil, seine Möglichkeit, stets ein Goethe-Zitat in Rede oder Schrift einzuflechten, sondern auch seine Gabe zur exzellenten Verkürzung von Botschaften. Die Vorworte seiner Bücher sind ein leicht zugänglicher Beleg für diese These.

Seine Kunst zu reden konnten auch Auswärtige vielfach genießen. Unvergessen ist die mit Geist, Witz und Charme gespickte Damenrede auf der Frankfurter Tagung des Verbands der Hochschullehrer für Betriebswirtschaft von 1990 oder die Dankesrede am Abend nach der Ehrenpromotion in München. Sie wirkten wie aus dem Ärmel geschüttelt und waren mit Sicherheit akribisch vorbereitet.

In den Jahren nach der Emeritierung hat er die Kontakte zu seinen früheren Mitarbeitern nicht aufgegeben. Auch wenn er Besuche reduzierte, nutzte er neben stets sofort handschriftlich beantworteten Briefen das Telefon ausgesprochen gerne. Hier fand jeder Anrufer einen aufgeweckten, zugeneigten und interessierten Zuhörer vor, für den ein Gespräch von 50 oder 60Minuten nichts Ungewöhnliches war. 
Mit Adolf Moxter ist ein Großer unseres Fachs der Betriebswirtschaftslehre gegangen. Er hat in unermüdlicher und kraftzehrender Weise nicht nur, aber insbesondere die Gebiete der Rechnungslegungstheorie und -praxis sowie der Unternehmensbewertung befruchtet und erweitert. Das betraf gleichermaßen seine Lehre und ließ ihn bei allem die Bewältigung zahlreicher anderer Aufgaben nicht vergessen. Auch hier setzte er Maßstäbe. Es bleibt zu hoffen, dass die Früchte dieser erfolgreichen Anstrengungen weiter gedeihen und Anwendung finden. 